\title{
Clustering Phenomenon of Chaotic Circuits Coupled Symmetrically by Mutual Inductors
}

\author{
Yuta KOMATSU, Yoko UWATE and Yoshifumi NISHIO \\ Department of Electrical and Electronic Engineering, Tokushima University \\ 2-1 Minami-Josanjima, Tokushima, Japan \\ Phone:+81-88-656-7470, Fax:+81-88-656-7471 \\ Email: \{komatsu, uwate, nishio\}@ee.tokushima-u.ac.jp
}

\begin{abstract}
In this study, the clustering phenomenon observed from chaotic circuits coupled by mutual inductors is investigated. In order to analyze the phenomena, dependent variables corresponding to phases of solutions for subcircuits are introduced. By using this dependent variables, we make detailed investigation on statistical information of the clustering for a six subcircuit case, such as sojourn time, cluster types, and so on.
\end{abstract}

\section{INTRODUCTION}

Spatiotemporal phenomena observed from large-scale coupled chaotic networks have attracted many researchers' attention and have been studied strenuously by many researchers. The studies on coupled chaotic networks are classified into two categories. One is discrete-time systems, the other is continuous-time systems. A lot of studies have been carried out for discrete-time mathematical models [1][2]. However, there have been a few studies on continuous-time real physical systems such as electrical circuits [3][4].

In this study, we particularly focus on the clustering phenomenon observed from continuous-time real physical systems in spatiotemporal phenomena. We investigate the clustering observed from chaotic circuits coupled by mutual inductors in detail. By computer calculations, occurrences of the clustering and chaotic changes of a synchronization state are confirmed. In order to analyze the phenomena, dependent variables corresponding to phases of solutions for subcircuits are introduced. By using this dependent variables, we make detailed investigation on statistical information of the clustering for a six subcircuit case, such as sojourn time, cluster types, and so on.

\section{Circuit Model}

Figure 1 shows a circuit model. In the circuit, $N$ identical chaotic circuits are coupled symmetrically by mutual inductors. Each chaotic subcircuit is a symmetric version of the circuit model proposed by Inaba et al. [5]. It consists of three memory elements, one linear negative resistor and one nonlinear resistor, and is one of the simplest autonomous chaotic circuits.

First, in order to implement circuit experiments, we consider the simplified circuit model shown in Fig. 2. In the circuit, $N$ identical chaotic circuits are coupled by an inductor. Then, in order to implement computer calculations, we approximate the $i-v$ characteristics of the nonlinear resistor consisting of diodes by the following function.

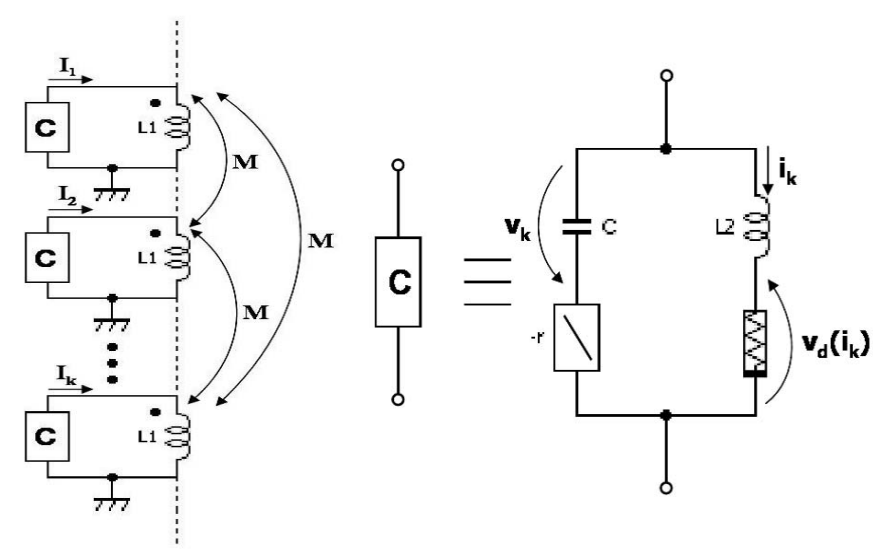

Fig. 1. Circuit model.

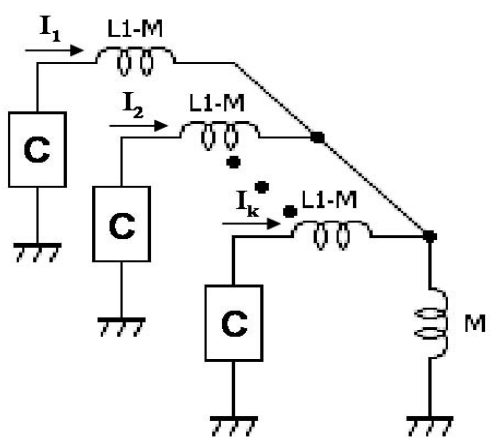

Fig. 2. Simplified circuit model.

$$
v_{d}\left(i_{k}\right)=\sqrt[9]{r_{d} i_{k}}
$$

By using the following variables and parameters,

$$
\begin{aligned}
& t=\sqrt{\left(L_{1}-M\right) C} \tau, \quad a=\sqrt[8]{r_{d} \frac{C}{L_{1}-M}}, \quad \cdots . "=\frac{d}{d \tau}, \\
& I_{k}=a \sqrt{\frac{C}{L_{1}-M}} x_{k}, \quad i_{k}=a \sqrt{\frac{C}{L_{1}-M}} y_{k}, \quad v_{k}=a z_{k}, \\
& \alpha=\frac{L_{1}-M}{L_{2}}, \quad \beta=r \sqrt{\frac{C}{L_{1}-M}}, \quad \gamma=\frac{M}{L_{1}},
\end{aligned}
$$


the circuit equations are normalized and described as

$$
\begin{aligned}
& \dot{x}_{k}=\beta\left(x_{k}+y_{k}\right)-z_{k} \\
&-\frac{\gamma}{1+(N-1) \gamma} \sum_{j=1}^{N}\left\{\beta\left(x_{j}+y_{j}\right)-z_{j}\right\} \\
& \dot{y}_{k}=\alpha\left\{\beta\left(x_{k}+y_{k}\right)-z_{k}-f\left(y_{k}\right)\right\} \\
& \dot{z}_{k}=x_{k}+y_{k} \quad(k=1,2, \cdots, N)
\end{aligned}
$$

where

$$
f\left(y_{k}\right)=\sqrt[9]{y_{k}} .
$$

In the following computer calculations, (3) is calculated by using the Runge-Kutta method with step size $\Delta t=0.005$.

\section{Clunstering Phenomenon}

We carried out computer calculations for the case of $N=$ 6. Figure 3 shows computer calculated results. From Fig. 3 , we can confirm occurrences of a clustering phenomenon and chaotic changes of a synchronization state. In Fig. 3(a), subcircuit 1 becomes in-phase synchronization state in almost all of the time and becomes asynchronous state for subcircuits 3 and 6 at moments. While, for subcircuits 2, 4, and 5, subcircuit 1 becomes anti-phase syncronization state in most intervals and becomes asynchronous state occasionally. As we can see from this figure, the cluster size of the cluster that subcircuit 1 is a component is principally 3 under these parameters.

By changing the parameter $\beta$, switching phenomena can be confirmed at $\tau=2500,5000$ as shown in Fig. 3(b). In the interval $[0,2500]$, subcircuit 1 is almost synchronized with subcircuits 2,4 and 5 at anti-phase, and almost becomes in-phase synchronization state for subcircuits 3 and 6. Moreover, the cluster size of the cluster that subcircuit 1 belongs to is principally 3 in this interval. While, subcircuit 1 becomes in-phase synchronization state for subcircuit 2 in the interval $[2500,5000]$ and for subcircuits 2 and 5 in the interval $[4000,5000]$. For subcircuits 3,4 and 6 , subcircuit 1 becomes anti-phase synchronization state in the interval $[2500,5000]$. In this interval, the cluster size for subcircuit 1 is principally 3 . Furthermore, subcircuit 1 is synchronized with subcircuits 3, 5 and 6 at anti-phase and becomes in-phase synchronization state for subcircuits 2 and 4 in almost all of the interval $[5000,10000]$. As the other intervals, the cluster size for subcircuit 1 is also principally 3 in this interval. Namely, we can consider that the cluster size for subcircuit 1 is easy to become 3 for the case of $N=6$.

\section{STATISTICAL ANALYSis}

\section{A. Introduction of Phase Variables}

In this section, we investigate the clustering phenomenon in detail. First, in order to discriminate whether each subcircuit is synchronized or not, let us define the Poincare section as $x_{1}<0$ and $z_{1}=0$. Next, we introduce the following independent variables from the discrete data of $x_{k}(n)$ and $z_{k}(n)$ on the Poincaré map. The values of $\varphi_{k}(n)$ correspond

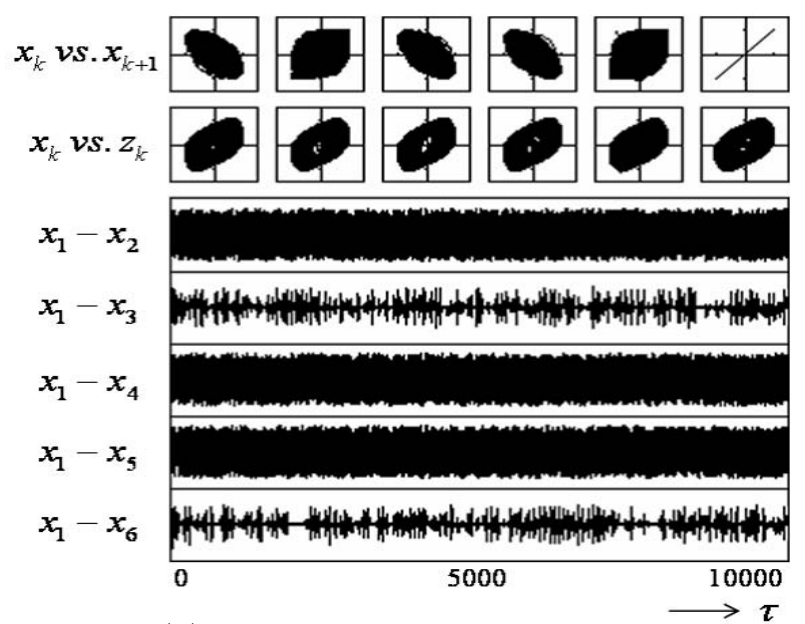

(a) $\alpha=20.0 . \beta=0.26 . \gamma=0.3$.

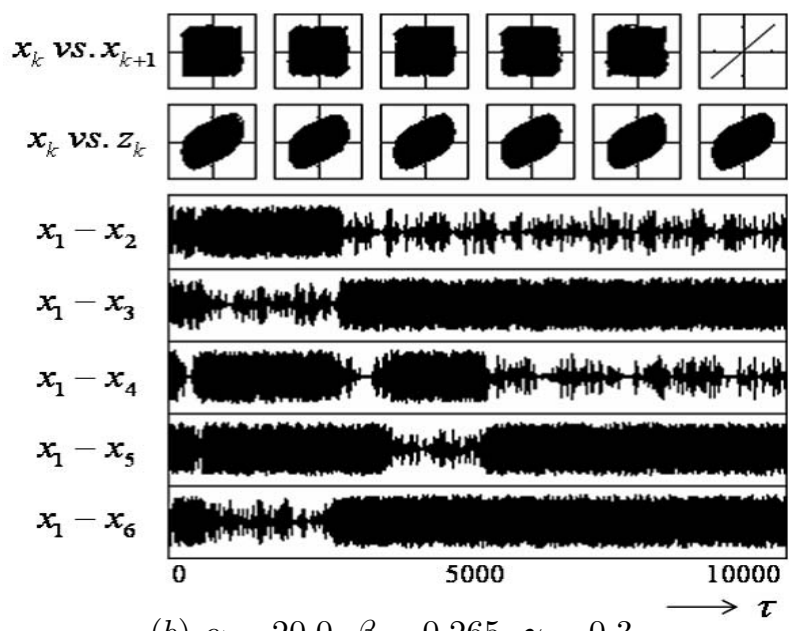

(b) $\alpha=20.0 . \beta=0.265 . \gamma=0.3$.

Fig. 3. Computer calculated results for $N=6 .(k=1,2,3,4,5,6$. $)$ $\left(x_{7}=x_{1}\right)$

to the phase differences between subcircuit 1 and the others. (Note that the argument of the point $\left(x_{1}(n), z_{1}(n)\right)$ is always $\pi$, because of the definition of the Poincaré map.) By using the values of $\varphi_{k}(n)$, we make detailed investigation on statistical information of the clustering for the case of $N=6$. In the statistical analysis, we carried out computer simulations during one hundred thousand iterations of the Poincaré map under the following parameters; $\alpha=20.0, \beta=0.265$, and $\gamma=0.3$.

$$
\varphi_{k}(n)=\left\{\begin{array}{l}
\pi-\tan ^{-1} \frac{z_{k}(n)}{x_{k}(n)} \\
x_{k}(n) \geq 0 \\
-\tan ^{-1} \frac{z_{k}(n)}{x_{k}(n)} \\
x_{k}(n)<0 \text { and } z_{k}(n) \geq 0 \\
2 \pi-\tan ^{-1} \frac{z_{k}(n)}{x_{k}(n)} \\
x_{k}(n)<0 \text { and } z_{k}(n)<0 \\
(k=2,3,4,5,6 .)
\end{array}\right.
$$




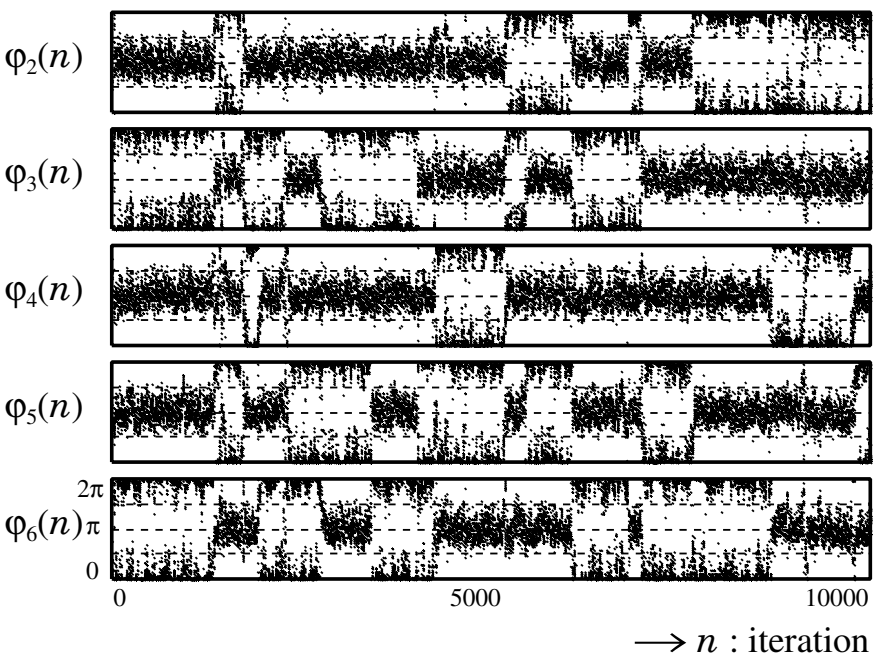

Fig. 4. Time series $\varphi_{k}(n)$ calculated from the data $x_{k}(n)$ and $z_{k}(n)$.

\section{B. Probability distribution of $\varphi_{k}(n)$}

Figure 4 shows the time evolution of $\varphi_{k}(n)$ calculated from the data $x_{k}(n)$ and $z_{k}(n)$. Horizontal axis is the iteration of the Poincare map. Vertical axis is the phase difference of each subcircuit for subcircuit 1 . Switching phenomena occur at short intervals and a synchronization state changes chaotically. Moreover, we can see that subcircuit 1 becomes in-phase or anti-phase synchronization state for five others with high frequency.

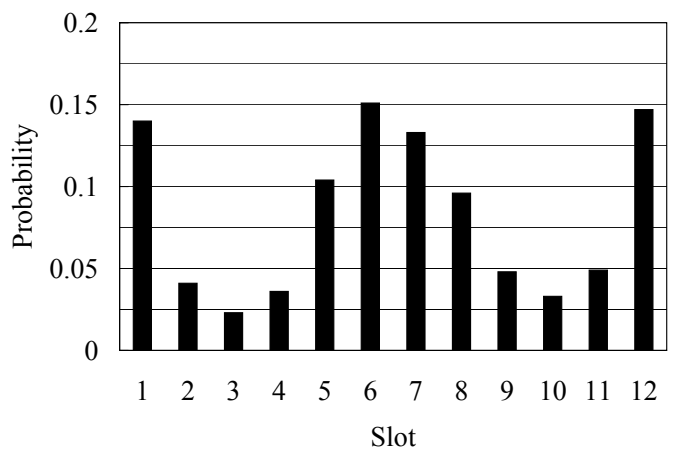

Fig. 5. Probability distributions of $\varphi_{2}(n)$. Horizontal slot $m$ indicates interval $[(m-1) \pi / 6, m \pi / 6]$.

Figure 5 shows the probability distributions of $\varphi_{2}(n)$. The probabilities of slots $1,6,7$, and 12 are larger than the others. We can consider that the slots 1 and 12 correspond to inphase states and the slots 6 and 7 correspond to anti-phase states. This means that each subcircuit becomes in-phase or anti-phase synchronization state with large probability. Furthermore, we have confirmed that the probability distributions of $\varphi_{k}(n)$ become about the same probability distributions. For the reason to become in this way, our circuit model's symmetry can be considered.

\section{Probability distributions of sojourn time}

We define that subcircuit 1 is synchronized with subcircuit $k$ if $\varphi_{k}(n)$ satisfies (6). This definition of the synchronization corresponds to the in-phase synchronization state.

$$
-\frac{\pi}{6}<\varphi_{k}(n)<\frac{\pi}{6} .
$$

Table I shows the probability distribution of the cluster size of the cluster that subcircuit 1 is a component. "Cluster size $=1$ " means that subcircuit 1 is not synchronized with the others. Moreover, "Cluster size $=2$ " means that subcircuit 1 is synchronized with one of five others and not synchronized with the others. As we can see from Tab. I, the probability of "Cluster size $=3$ " is the largest and the cluster size hardly becomes more than 4 .

TABLE I

PROBABILITY DISTRIBUTION OF THE CLUSTER SIZE FOR SUBCIRCUIT 1.

\begin{tabular}{|c|c||c|c|}
\hline Cluster size & Probability & Cluster size & Probability \\
\hline \hline 1 & 0.18869 & 4 & 0.00020 \\
\hline 2 & 0.30057 & 5 & 0.00000 \\
\hline 3 & 0.51054 & 6 & 0.00000 \\
\hline
\end{tabular}

While, Fig. 6 shows the probability distributions of sojourn time. The slots in the horizontal axis of the figure denote the ranges of the sojourn time and are summarized in Tab. II. For example, compared Fig. 6(b) with Fig. 6(c), the probability distributions of the slots 3,4 , and 5 of "Cluster size $=2$ " are larger than those of "Cluster size $=3$ ". Furthermore, "Cluster size $=3$ " disappears within 1 or 2 iterations of the Poincaré map. Clearly, it is confirmed that the larger cluster size is, the larger sojourn time is as shown in Fig. 6, except the case of "Cluster size $=4 "$.

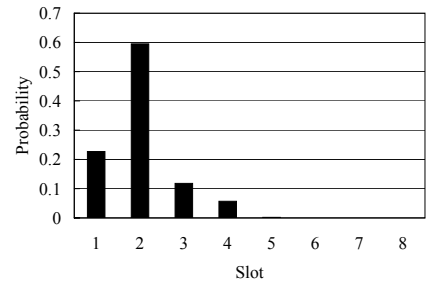

(a) Cluster size $=1$.

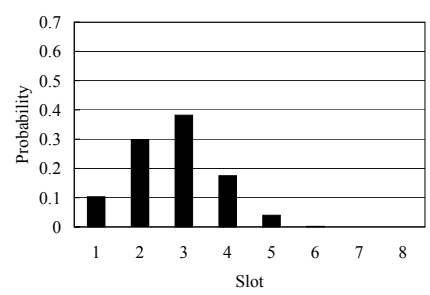

(c) Cluster size $=3$.

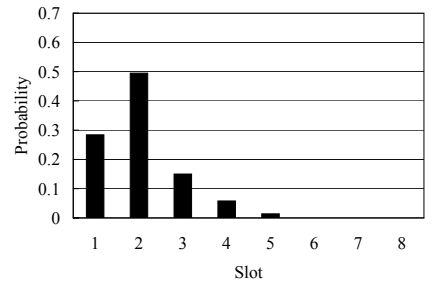

(b) Cluster size $=2$.

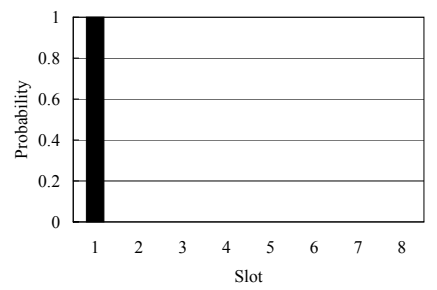

(d) Cluster size $=4$.
Fig. 6. Probability distributions of sojourn time.

TABLE II

RANGES OF SLOTS IN FIG. 5.

\begin{tabular}{|c|c||c|c|}
\hline Slot & Sojourn time $(n)$ & Slot & Sojourn time $(n)$ \\
\hline \hline 1 & $1-2$ & 5 & $21-50$ \\
2 & $3-5$ & 6 & $51-100$ \\
3 & $6-10$ & 7 & $101-200$ \\
4 & $11-20$ & 8 & $201-500$ \\
\hline
\end{tabular}




\section{Probability distribution of various cluster types.}

The probability distribution of specific cluster types for subcircuit 1 is shown in Tab. III. For example, the probability of the cluster type "1-2-3-4-5-6" is $2.04 \times 10^{-3}$. This cluster type means that all subcircuits become asynchronous states and the number of clusters is 6 . Then, we can confirm that the probability of the cluster type "12-3-4-5-6" is $1.89 \times 10^{-3}$. This cluster type means that subcircuit 1 is syncronized with subcircuit 2 and the others are not synchronized and the number of clusters is 5. Moreover, the cluster type "12-34-5-6" means that subcircuit 1 is synchronized with subcircuits 2 and subcircuit 3 is synchronized with subcircuits 4 and subcircuits 5 and 6 independently oscillate and the number of clusters is 4.

While, the probability distribution of cluster types is shown in Tab. IV. For example, the probability of the cluster type "2$1-1-1-1$ " is $3.06 \times 10^{-2}$. This cluster type means that any two subcircuits are synchronized and the others are asynchronous states and the number of clusters is 5. Furthermore, the cluster type "2-2-1-1" means that any two subcircuits are synchronized and one cluster is formed and any two others of four subcircuits are synchronized and another cluster is also formed and the others are independently oscillate.

\section{DISCUSSION}

First, we considered the probability distribution of specific cluster types shown in Tab. III as experimental values. Then, we considered that the statistical data shown in Tab. IV could be calculated as theoretical figures only by using the information shown in Tab. III. For example, the probability $P_{1}$ of the cluster type "2-1-1-1-1" can be calculated by using the probability of the cluster type "12-3-4-5-6" as follows.

$$
P_{1}={ }_{5} C_{1} \times 0.00189=9.45 \times 10^{-3} \text {. }
$$

As the same way, the probability $P_{2}$ of the cluster type "2-2$1-1$ " can be calculated by using the probability of the cluster type "12-34-5-6" as follows.

$$
P_{2}={ }_{5} C_{1} \times{ }_{4} C_{2} \times 0.00359=0.1077 .
$$

Moreover, the other various probabilites can be calculated by using the probability information shown in Tab. III. These results are summarized in Tab. V.

The relative error between experimental values and theoretical figures for cluster types is shown in Tab. VI. We can confirm that the relative errors of the cluster types "2-1-1-1-1", "2-2-2", "3-1-1-1", "4-1-1", and "4-2" are relatively large. We have to investigate a reason to become in this way in detail. However, if we could calculate various probabilites shown in Tab. IV by using a little information shown in Tab. III, it would be quite effective for the analysis when a circuit scale is large.

\section{CONCLUSIONS}

In this study, we investigated the clustering phenomenon observed from chaotic circuits coupled by mutual inductors. By carrying out computer calculations, we confirmed occurrences of the clustering and chaotic changes of a synchronization state for a six subcircuit case. Moreover, we made detailed investigation on statistical information of the clustering, such as sojourn time, cluster types, and so on.

\section{ACKNOWLEDGEMENTS}

This work was partly supported by Yazaki Memorial Foundation for Science and Technology.

\section{REFERENCES}

[1] K. Kaneko, "Clustering, Coding, Switching, Hierarchical Ordering, and Control in a Network of Chaotic Elements," Physica D, vol.41, pp.137$172,1990$.

[2] Y. Maistrenko, O. Popovych and M. Hasler, "On Strong and Weak Chaotic Partial Synchronization," Int. J. Bifurcation Chaos, vol.10, no.1, pp.179203, 2000.

[3] M. Miyamura, Y. Nishio and A. Ushida, "Clustering in Globally Coupled System of Chaotic Circuits," Proc. of ISCAS'02, vol. 3, pp.57-60, May 2002.

[4] Y. Nishio and A. Ushida, "Chaotic Wandering and its Analysis in Simple Coupled Chaotic Circuits" IEICE Transactions on Fundamentals, vol E85-A, no. 1, pp. 248-255, Jan. 2002.

[5] N. Inaba and S. Mori, "Chaotic Phenomena in Circuits with a Linear Negative Resistance and an Ideal Diode," Proc. of MWSCAS'88, pp.211214, Aug. 1988.

TABLE III

PROBABILITY DISTRIBUTION OF SPECIFIC CLUSTER TYPES FOR SUBCIRCUIT 1.

\begin{tabular}{|c|c||c|c|}
\hline Cluster type & Probability & Cluster type & Probability \\
\hline \hline $1-2-3-4-5-6$ & 0.00204 & $123-456$ & 0.02845 \\
\hline $12-3-4-5-6$ & 0.00189 & $1234-5-6$ & 0.00001 \\
\hline $12-34-5-6$ & 0.00359 & $1234-56$ & 0.00002 \\
\hline $12-34-56$ & 0.00055 & $12345-6$ & 0.00000 \\
\hline $123-4-5-6$ & 0.00467 & 123456 & 0.00000 \\
\hline $123-45-6$ & 0.01491 & & \\
\hline
\end{tabular}

TABLE IV

PROBABILITY DISTRIBUTION OF CLUSTER TYPES.

\begin{tabular}{|c|c||c|c|}
\hline Cluster type & Probability & Cluster type & Probability \\
\hline \hline $1-1-1-1-1-1$ & 0.00204 & $3-3$ & 0.25662 \\
\hline $2-1-1-1-1$ & 0.03006 & $4-1-1$ & 0.00033 \\
\hline $2-2-1-1$ & 0.10004 & $4-2$ & 0.00048 \\
\hline $2-2-2$ & 0.00597 & $5-1$ & 0.00000 \\
\hline $3-1-1-1$ & 0.08581 & 6 & 0.00000 \\
\hline $3-2-1$ & 0.51865 & & \\
\hline
\end{tabular}

TABLE V

THEORETICAL FIGURE OF THE PROBABILITY DISTRIBUTION OF CLUSTER TYPES.

\begin{tabular}{|c|c||c|c|}
\hline Cluster type & Probability & Cluster type & Probability \\
\hline \hline $1-1-1-1-1-1$ & 0.00204 & $3-3$ & 0.28450 \\
\hline $2-1-1-1-1$ & 0.00945 & $4-1-1$ & 0.00010 \\
\hline $2-2-1-1$ & 0.10770 & $4-2$ & 0.00020 \\
\hline $2-2-2$ & 0.01650 & $5-1$ & 0.00000 \\
\hline $3-1-1-1$ & 0.04670 & 6 & 0.00000 \\
\hline $3-2-1$ & 0.44730 & & \\
\hline
\end{tabular}

TABLE VI

RELATIVE ERROR BETWEEN EXPERIMENTAL VALUES AND THEORETICAL FIGURES FOR CLUSTER TYPES.

\begin{tabular}{|c|c||c|c|}
\hline Cluster type & Relative error & Cluster type & Relative error \\
\hline \hline $2-1-1-1-1$ & 2.18095 & $3-2-1$ & 0.15951 \\
\hline $2-2-1-1$ & 0.07112 & $3-3$ & 0.09800 \\
\hline $2-2-2$ & 0.63818 & $4-1-1$ & 2.30000 \\
\hline $3-1-1-1$ & 0.83747 & $4-2$ & 1.40000 \\
\hline
\end{tabular}

